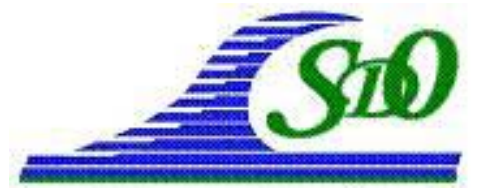

XI ${ }^{\text {èmes }}$ Journées Nationales Génie Côtier - Génie Civil

Les Sables d'Olonne, 22-25 juin 2010

DOI:10.5150/jngcgc.2010.052-B @ Editions Paralia CFL

disponible en ligne - http://www.paralia.fr - available online

\title{
Reconnaissance de menhirs subtidaux par sonar latéral et bathymetrie fine en baie de Quiberon
}

\author{
Agnès BALTZER ${ }^{1}$, Chantal BONNOT-COURTOIS ${ }^{2}$, Serge CASSEN $^{3}$, \\ Jérôme FOURNIER ${ }^{4}$, André LORIN ${ }^{5}$, Renaud CAGNA ${ }^{6}$, Arnaud GILLIER ${ }^{6}$
}

1. Université de Caen, Laboratoire Morphodynamique Continentale et Côtière, UMR 6143 M2C. 14000 Caen, France.

agnes.baltzer@unicaen.fr

2. UMR 8586 PRODIG CNRS, Laboratoire de Géomorphologie et Environnement

Littoral. 15, bvd de la mer, 35800 Dinard, France. chantal.bonnot@ephe.sorbonne.fr

3 UMR 6566, Université de Nantes, Laboratoire de Recherche Archéologiques, BP

81227 , 44312 Nantes Cedex 3, France.serge.cassen@univ-nantes.fr

4. Muséum d'Histoire Naturelle de Dinard, UMR BOREA 8153, Rue du Port Blanc, 35800 Dinard, France.jfournier@mnhn.fr

5. GRHASM, 44 700, Orvault, France. alorin@free.fr

6. GENAVIR, Zone industielle de la Pointe du Diable, 29280 Plouzané, France.

haliotis@genavir.fr

\section{Résumé :}

Sur la côte Est de la presqu'île de Quiberon, Kerbougnec et le Beg Rohu sont deux gisements néolithiques sous-marins extraordinaires par la qualité et l'ampleur des découvertes faites ces dernières années. Toute la question repose sur l'expansion supposée des deux sites, voisins de quelques centaines de mètres, notamment en direction du large. Des vestiges (menhirs et stèles d'une longueur variant de $80 \mathrm{~cm}$ à $2 \mathrm{~m}$ ) ont été reconnus dans les deux sites jusqu'à la limite des plus basses eaux. Pour accéder au domaine immergé, plusieurs campagnes de sonar latéral et une campagne de bathymétrie fine (sondeur multifaisceaux de la vedette Haliotis de l'Ifremer) ont été réalisées entre 2006 et 2009 afin d'identifier, dans un premier temps, la signature acoustique du "menhir marin" puis de réaliser une cartographie de ces objets.

Cette cartographie sous-marine conduit à délimiter l'extension des menhirs submergés, et par là même à retrouver la limite du paléo-rivage au Néolithique, sachant que ces menhirs étaient érigés sur la terre ferme dès 6800 ans BP. La comparaison des données d'imagerie sonar et de bathymétrie, permet d'évaluer les avantages et inconvénients de ces deux approches. La bathymétrie très fine semble être un outil particulièrement intéressant pour les reconnaissances archéologiques, car elle permet de "voir" directement le relief, même peu marqué des objets étudiés. Ceci n'est pas le cas pour l'imagerie sonar qui fournit surtout une information sur la nature du fond. Ainsi, un bloc mégalithique posé sur le platier rocheux apparaîtra avec le même faciès acoustique et sera donc difficile à distinguer du substratum. A l'inverse, toutes les structures posées 
ou rajoutées dans un environnement naturel vont se traduire par des irrégularités morphologiques et donc bathymétriques parfaitement visibles à l'échelle du sondeur multifaisceaux de l'Haliotis.

\section{Mots clefs :}

Menhirs - Niveau marin - Sonar latéral et interférométrique - Bathymétrie Néolithique

\section{Introduction}

La détection des variations holocène du niveau marin s'effectue de manière privilégiée sur le domaine littoral, lieu des interactions océan/continent, à travers l'analyse des enregistrements sédimentaires par les géologues. Il apparaît toutefois particulièrement difficile d'établir un cadre chronologique précis de la remontée du niveau marin depuis le dernier maximum glaciaire (18000 ans BP).

L'archéologie rend possible l'établissement de ce cadre chronologique de façon précise depuis 10000 ans BP grâce à l'étude de l'activité humaine (GIOT et al., 1979 ; BAILLOUD et al., 1995; GAUDIN, 2004). En effet, cette activité réagit aux modifications de l'environnement naturel littoral, qui sont directement liées aux variations du niveau marin. A partir de cette double approche, notre étude se propose de suivre les variations du niveau marin holocène dans la Baie de Quiberon lors de l'ennoiement de la plateforme sud armoricaine en s'appuyant sur l'analyse des différentes traces de l'activité humaine aux temps préhistoriques telles que les pierres dressées ou les stèles. Ainsi la connaissance archéologique permettrait de répondre aux questions du géologue quant à la chronologie des évènements.

Le Sud de la Bretagne et plus particulièrement le Golfe du Morbihan et la Baie de Quiberon (figure 1) semble être, grâce aux travaux archéologiques de CASSEN et VAQUERO LASTRES (2003), CASSEN et al. (2008), CASSEN et al. (2010) et BOUJOT et PINET (2007), un lieu d'étude particulièrement favorable. En effet, ces auteurs soulignent que la compréhension et la connaissance de l'architecture symbolique, émergeant à la période de transition située entre le Mésolithique et le Néolithique, nécessitent une représentation dans l'espace des différents alignements de pierres et stèles. Par exemple, le site du Moulin à Kerbougnec (Saint Pierre de Quiberon) s'avère représenter un énorme complexe de plus d'une dizaine d'alignements de stèles orientées sur des points d'affleurement, sur plusieurs centaines de mètres le long d'un développement architectonique dont l'extrémité (en permanence submergée) n'est toujours pas reconnue.

C'est sur le site voisin du Petit Rohu, situé à quelques centaines de mètres, qu'ont été trouvées deux couples de lames de haches en jadéitite alpine à l'été 2007 (CASSEN et al., 2010).

La première phase de notre étude porte sur l'analyse fine des données acoustiques dans un plan horizontal, en essayant de tirer le maximum d'information de l'imagerie sonar, 


\section{XI ìmes Journées Nationales Génie Côtier-Génie Civil}

Les Sables d'Olonne, 22-25 juin 2010

des plongées, des photos à marée basse et des données bathymétriques afin d'utiliser les mégalithes submergés comme marqueurs du paléo-rivage néolithique, permettant ainsi de la cartographier.

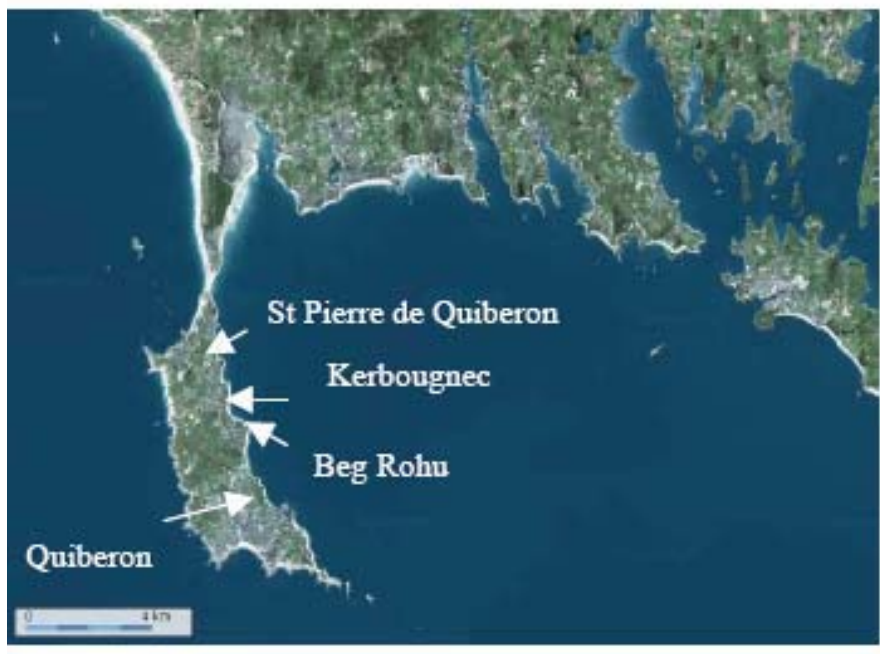

Figure 1. Localisation des sites mégalithiques submergés de Kerbougnec et du Petit Rohu.

\section{Méthodologie}

Cinq campagnes de sonar latéral associées à des validations par des plongées ont été réalisées entre 2005 et 2009 afin d'identifier la signature acoustique de mégalithes submergés ou "menhirs marins" puis de réaliser une cartographie de ces objets. L'ensemble des profils sonar a été superposé à des mosaïques de photographies aériennes géoréférencées (Ortholittorale 2000, www.geolittoral.equipement.gouv.fr) à l'aide d'un logiciel de Système d'Information Géographique (SIG, ArcGIS). L'analyse d'image a permis de localiser les anomalies acoustiques susceptibles d'être des mégalithes. La qualité des photos aériennes permet en effet de distinguer des alignements de mégalithes à terre. En les prolongeant en direction de la mer, il est ainsi possible de "retrouver" les alignements sous-marins (figure 2).

Nous avons utilisé 3 types de sonars latéraux qui se caractérisent par une fréquence d'émission différente, sachant que plus la fréquence est haute, plus la résolution de l'image est précise.

\section{A. Le sonar à balayage latéral Edgetech $272 \mathrm{TD}$ (fréquence $100 \mathrm{kHz}$ )}

Le sonar latéral Edgetech 272 TD présente les caractéristiques suivantes : une longueur de $1,20 \mathrm{~m}$ pour un poids de $25 \mathrm{~kg}$. Il possède 2 fréquences d'émission (100 et $500 \mathrm{kHz}$ ) ce qui signifie peu ou pas de pénétration du signal pour une résolution horizontale de 40 cm dans des conditions optimales. Pour les reconnaissances classiques, il est utilisé avec 
Thème 3 - Instrumentation, mesures, imagerie et télédétection

une fréquence de $100 \mathrm{kHz}$ (permet de déceler des objets ou structures sous quelques $\mathrm{cm}$ de sédiments) à une altitude d'environ $10 \mathrm{~m}$ au dessus du fond. Cette configuration permet l'acquisition d'une image acoustique de $100 \mathrm{~m}$ de large de chaque côté donc une bande totale insonifiée, dite fauchée, de $200 \mathrm{~m}$ de large. L'acquisition de ce type de données se fait à l'aide du logiciel Isisonar (de TritonElics), avec un positionnement en temps réel intégré à partir dun GPS. Le traitement de l'image a posteriori et sa visualisation dans un système géographique se fait par l'intermédiaire du logiciel Delphmap (de TritonElics). Ce type de données a déjà été utilisé en archéologie (ATALLAH et al., 2005)

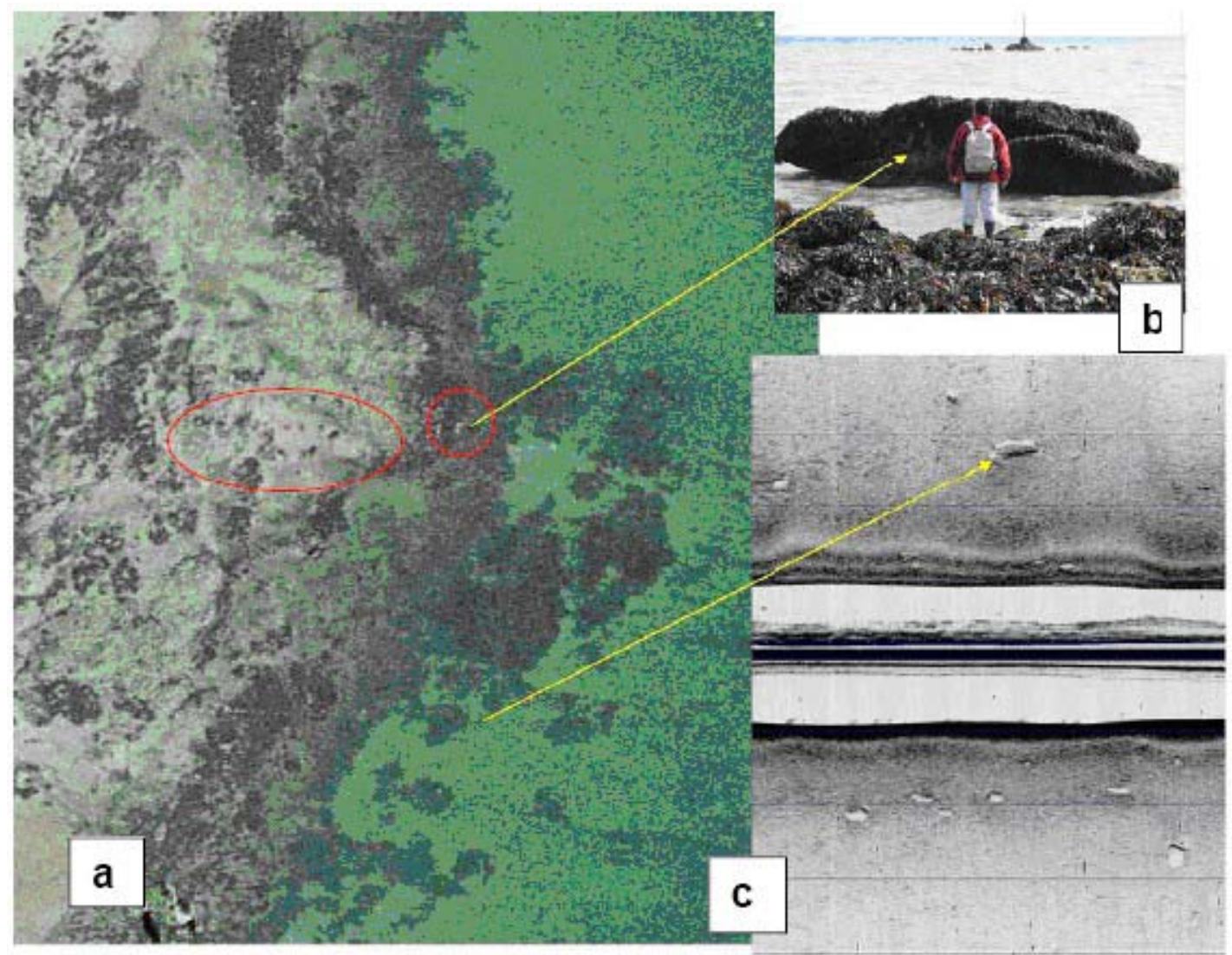

Figure 2. Site de Kerbougnec. a : Photo aérienne montrant 3 alignements découverts à marée basse dans le cercle rouge. b : Grande stèle émergée à marée basse . $c$ : Mégalithes identifiés sous l'eau par le sonar latéral (non visibles sur la photo aérienne).

\section{B. Le sonar à balayage latéral bi-canal SH1 dit Sture Hultqvist (fréquence $500 \mathrm{kHz}$ )}

Ce sonar prototype a été construit par un suédois, Mr Sture Hultqvist (décédé en 2007) qui n'a pas donné de nom spécifique à cet outil. D'un encombrement plus réduit que le modèle précédent, il sert essentiellement à Mr André Lorin pour l'exploration des épaves antiques et historiques. Ce sonar pèse $7 \mathrm{~kg}$ pour une longueur de $95 \mathrm{~cm}$, avec 


\section{XI $I^{\text {èes }}$ Journées Nationales Génie Côtier - Génie Civil \\ Les Sables d'Olonne, 22-25 juin 2010}

une fréquence d'émission 200 ou $500 \mathrm{kHz}$ suivant la sonde amovible utilisée. Il possède une résolution horizontale de $10 \mathrm{~cm}$ environ pour le modèle à $500 \mathrm{kHz}$. Utilisé en pratique avec une largeur de bande de 25 à $125 \mathrm{~m}$ de chaque côté. Les images obtenues avec le logiciel Sub 277 sont sous format XTF. Le post-traitement de ces images s'est fait également avec Isisonar et Delphmap de Triton Elics (figure 2).

\section{Le sonar interférométrique de l'Haliotis (Geoswath de Geoacoustic) (Fréq. $250 \mathrm{kHz}$ )}

Ce sonar interférométrique, fixé sous la coque de la vedette Haliotis de l'Ifremer (opérationnelle depuis mai 2008), conçue pour naviguer et opérer dans des petits fonds, de $2 \mathrm{~m}$ à $20 \mathrm{~m}$. Petite embarcation de $10 \mathrm{~m}$ pour un tirant d'eau de $0,5 \mathrm{~m}$, elle est équipée d'outils acoustiques sophistiqués et complémentaires permettant de faire des reconnaissances très précises de la zone côtière. Le sondeur interférométrique (ou Geoswath) fonctionne sur une fréquence de $250 \mathrm{kHz}$. Il présente la particularité de pouvoir acquérir simultanément une image acoustique (type sonar) ainsi qu'un bathymétrie fine, avec une résolution inférieure au mètre. Sa portée est de 10 fois la profondeur d'eau en mode imagerie et 5 fois la profondeur d'eau en mode bathymétrie. Il faut cependant noter que le traitement de ces données ne peut se faire correctement qu'avec des logiciels propres au constructeur ou à l'Ifremer.

\section{Résultats}

L'ensemble des structures architecturales de type "alignement" a été inséré au sein d'un Système d'Information Géographiqe (sous ArcGIS). Le même projet géoréférencé recense l'ensemble des sonogrammes, des orthophotographies littorales, des points d'anomalies inventoriés et la bathymétrie.

Les différents types de profils sonar acquis pendant ces missions (figures 3 et 4) ont été projetés sur l'ortholittorale 2000-2002 à l'échelle du 1:25000 (NTF Clarke 1880, Lambert II étendu). Les sonogrammes Mos1545b et Mos1549a (figure 3) montrent clairement des limites de trois zones de faciès acoustiques homogènes :

- Une zonetrès foncée de l'image présentant une forte réflectivité du fond qui correspond à la plage de sable visible sur la photo aérienne;

- Une zone plus claire (gris moyen), de réflectivité moyenne, correspondant probablement à un sédiment de type sable fin à moyen qui rétrodiffuse une grande partie du signal;

- Une zone de faible réflectivité (apparaissant en gris très clair sur l'image sonar), en bas de plage, qui est là aussi parfaitement visible et délimitée d'une part par la plage et d'autre part par le faciès gris moyen. Ce faciès très clair correspond probablement soit à des sédiments plus fins (type vase) soit à des niveaux très "absorbants" d'un point de vue acoustique, de type tourbe. On retrouve donc une succession classique de la plage vers le bas estran de sable, sédiments vaseux (ou tourbe), et apparition de faune et flore en bas estran. 
La figure 4 restitue le profil Mos028bis acquis à l'aide du sonar SH1 et révèle clairement l'architecture des stèles qui se distingue du signal donné par les graviers et tourbes dégagés sur la partie gauche du sonogramme (Mos1549a - figure 3).

La résolution de l'image est nettement meilleure: il est possible de délimiter précisément chaque roche de l'ensemble des stèles. Les haches en jadéite et la lame polie en fibrolite qui ont été découvertes (voir figure 4) et les stèles se retrouvent dans chaque cas sur la limite de deux faciès, en bordure du faciès gris très clair. Il pourrait être intéressant de suivre cette limite pour voir si l'on ne trouve pas d'autres affleurements. La synthèse de tous les travaux réalisés dans cette zone, en partant de la terre jusqu'à la zone des $-8 \mathrm{~m}$ montre l'existence de plusieurs alignements de mégalithes, qui ont été authentifiés lors de la dernière plongée (octobre 2009).

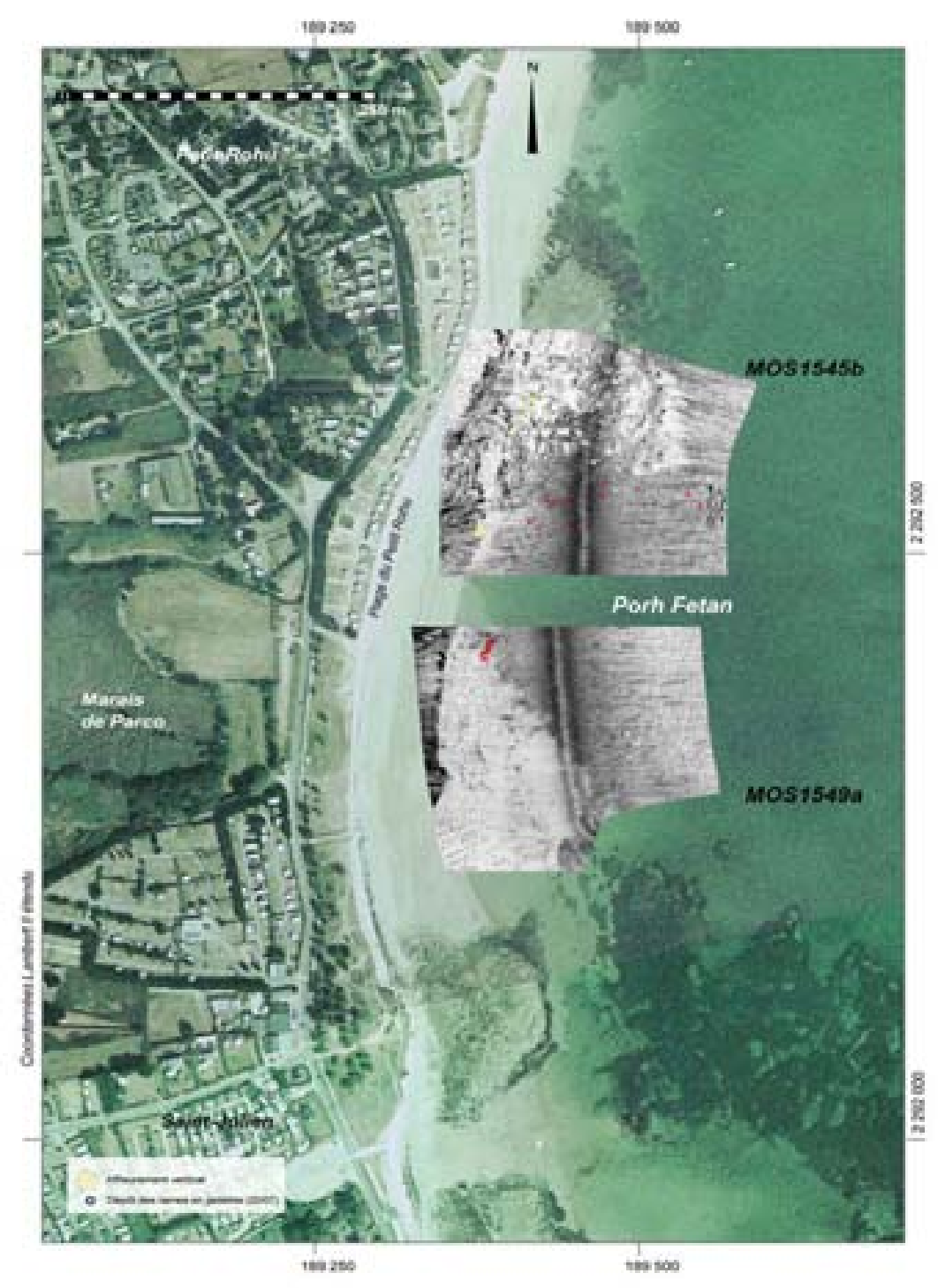

Figure 3. Exemple de sonogrammes acquis avec l'Edgetech 272 TD sur le site du Porh Fetan. 


\section{XI ìmes Journées Nationales Génie Côtier-Génie Civil}

Les Sables d'Olonne, 22-25 juin 2010
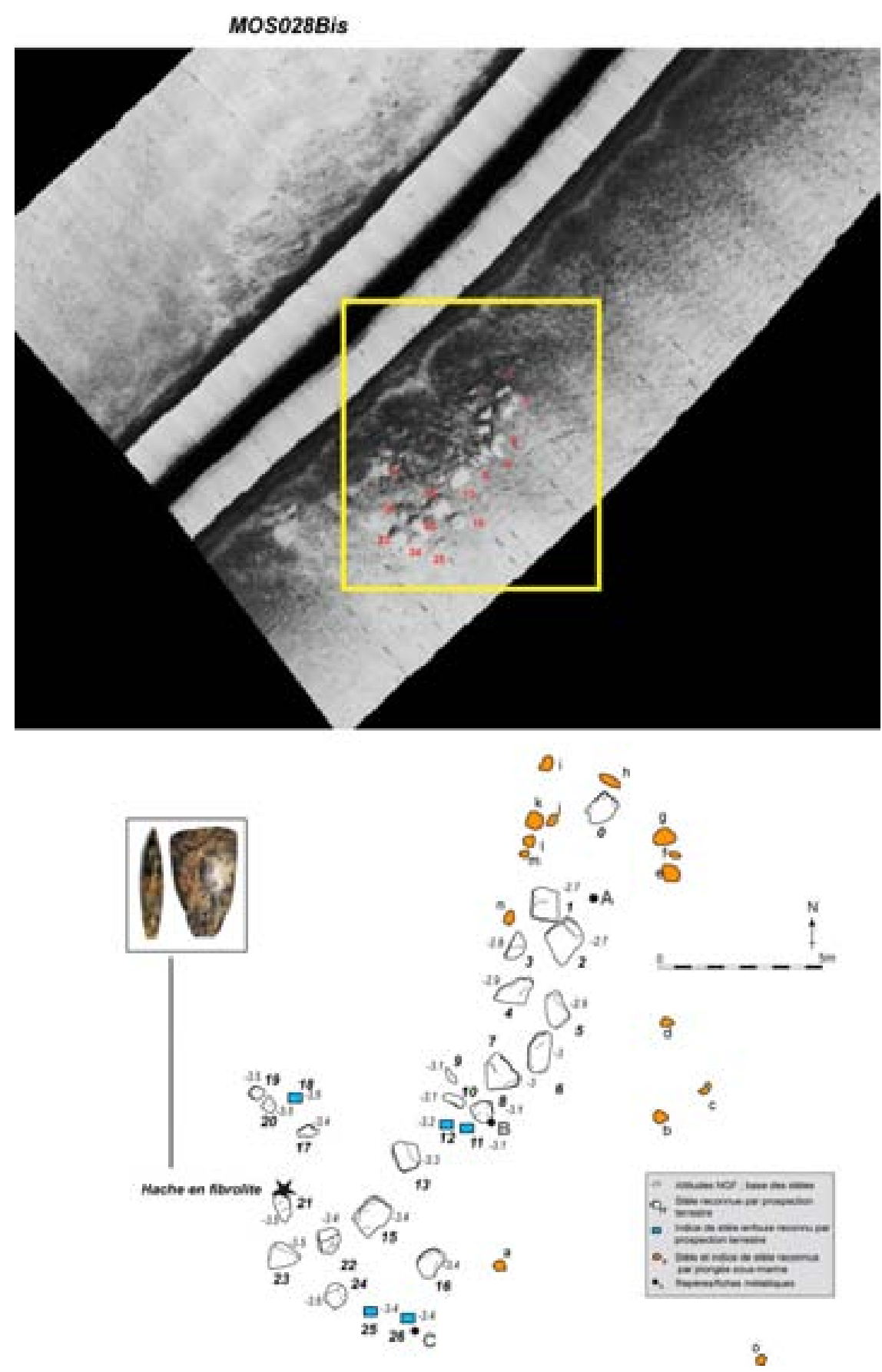

Figure 4. Sonogramme acquis avec le sonar SH1 sur la barre de stèles à Porh Fetan.

En particulier, l'alignement de menhirs Ouest/Est passant par les points localisés par un GPS, 105 et 108 est confirmé. Les alignements se prolongeraient ainsi sur plus de $500 \mathrm{~m}$ de longueur, de la terre à la mer. Au-delà de la ligne bathymétrique des $-3 \mathrm{~m}$ (soit 
Thème 3 - Instrumentation, mesures, imagerie et télédétection

environ "-6 m" IGN69), aucune stèle n'ont été découverts. Il est donc possible d'émettre l'hypothèse que le paléo-rivage néolithique se situerait au niveau de cette limite.

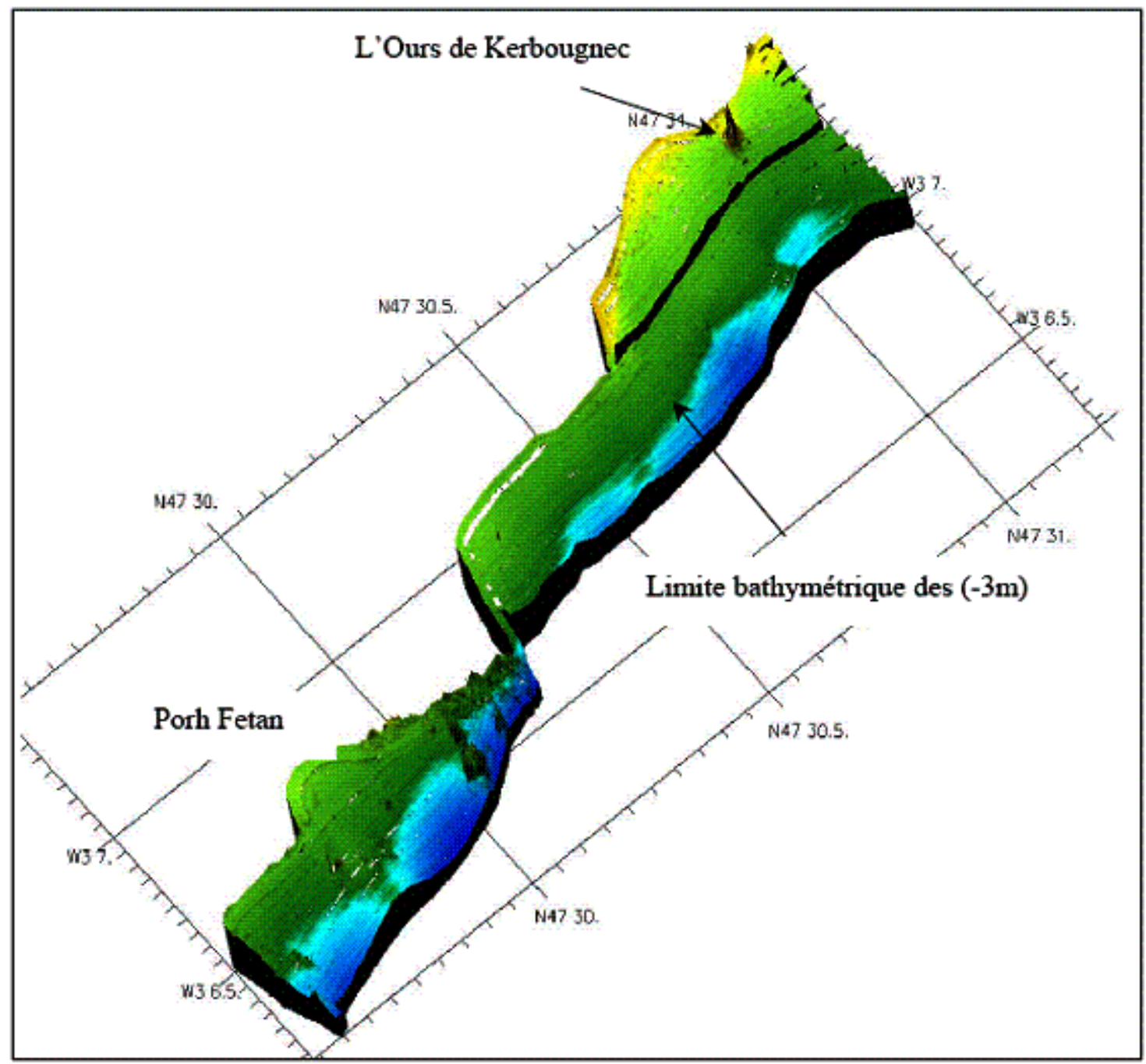

Figure 5. Carte bathymétrique obtenue à partir des données du sonar interférométrique de l'Haliotis sur la zone étudiée.

L'analyse détaillée de l'imagerie acoustique met en évidence la difficulté de différencier une stèle (bloc allochtone) sur le platier rocheux local. Afin d'essayer de lever ce doute, la Mission Mégaquib a été programmée du 22 au 29 Mai 2009 sur la vedette de l'Ifremer : l'Haliotis. Cette mission a permis d'utiliser pour la première fois le sonar interférométrique pour effectuer une reconnaissance archéologique.

Cette approche semble très prometteuse bien que les données ne soient pas encore totalement traitées. On peut cependant constater que la résolution de la bathymétrie, au travers de la micro-rugosité du fond, va permettre de "voir" les roches posées sur le fond. En effet, la sensibilité du sonar interférométrique permet d'enregistrer tous les 
échos, même ceux issus d'objets de taille inférieure à $30 \mathrm{~cm}$. Le traitement de ce type de donnée reste cependant délicat, car l'invalidation des sondes est faite manuellement : une certaine expérience semble donc nécessaire pour être capable de traiter correctement les données.

\section{Conclusions}

Si le sonar à balayage latéral s'avère être un outil tout à fait pertinent pour le repérage et la visualisation des menhirs subtidaux, le sonar interférométrique de coque permet en sus de les positionner avec une très grande précision $(<10 \mathrm{~cm})$. Ainsi la limite d'extension géographique maximum des menhirs immergés dans la zone de Kerbougnec permet de retrouver la position probable du paléo-rivage néolithique autour $\mathrm{du}-5 \mathrm{~m}$ NGF. L'exploitation de ce type de données issues de la prospection géophysique apporte de précieuses informations pour les recherches archéologiques en contexte sous-marin.

\section{Références bibliographiques}

ATALLAH L., SHANG C., BATES R. (2005). Object detection at different resolution in archaeological side-scan sonar images. Oceans - Europe, Volume 1, Issue 20-23, pp 287-292. doi:10.1109/OCEANSE.2005.1511727

BAIllOUd G., BOUJOT C., CASSEN S., LE ROUX C.-T. (1995). Carnac. Les Premières architectures de pierre. Paris, Éd. du CNRS, CNMHS (Patrimoine au présent), $126 \mathrm{p}$.

BOUJOT C., PINET L. (2007). Mégalithes et pierres dressées, matériau du discours scientifique en Préhistoire : évolution et perspectives d'après les exemples de Carnac (Bretagne) et du plateau de Cauria (Corse). In J. Évin dir., Congrès du centenaire de la S.P.F., Un siècle de construction du discours scientifique en Préhistoire, Vol. 3, pp 85-197.

CASSEN S., VAQUERO LASTRES J. (2003). Les Marches du Palais. Recherches archéologiques sur alignements de stèles et tertres funéraires néolithiques autour de la baie de Quiberon (Morbihan, 2000-2002). Nantes, Ed. Laboratoire de Préhistoire, Université de Nantes, 166 p., 135 fig., 4 pl coul.

CASSEN S., BOUJOT C., ERRERA M., MARGUERIE D., MENIER D., PAILLER Y., PÉTREQUIN P., POIRIER S., VEYRAT E., VIGIER E. (2008). Discovery of an underwater deposit of Neolithic polished axeheads and a submerged stone alignment at Petit Rohu near Saint-Pierre-Quiberon (Morbihan, France). Antiquity, Vol. 82, Issue 316.

CASSEN S., BOUJOT C., ERRERA M., MENIER D., PAILLER Y., PETREQUIN P., MARGUERIE D., VEYRAT E., VIGIER E., POIRIER S., DAGNEAU C., DEGEZ D., LORHO T., NEVEU-DEROTRIE H., OBELTZ C., SCALLIET F., SPARFEL Y. (2010). Un dépôt sous-marin de lames polies néolithiques en jadéitite et un ouvrage de 
Thème 3 - Instrumentation, mesures, imagerie et télédétection

stèles submergé sur la plage dite du Petit Rohu près Saint-Pierre-Quiberon (Morbihan). Bulletin de la Société Préhistorique Française, Vol. 107, pp 53-84.

GAUDIN L. (2004). Transformations spatio-temporelles de la végétation du nord-ouest de la France depuis la fin de la dernière glaciation. Reconstitutions paléo-paysagères. Thèse de doctorat, Mention: Archéologie et Archéométrie, Université de Rennes 1, UFR Structure et Propriétés de la Matière.

GIOT P.-R., L'HELGOUAC'H J., MONNIER J. (1979). Préhistoire de la Bretagne. Rennes : Ouest France Université, 1979, 444 p. 